\title{
Criminologie
}

\section{La judiciarisation des rébellions par les forces de l'ordre à Bruxelles de 1945 à 1975}

\section{Melpomeni Skordou}

Volume 45, numéro 2, automne 2012

URI : https://id.erudit.org/iderudit/1013728ar

DOI : https://doi.org/10.7202/1013728ar

Aller au sommaire du numéro

Éditeur(s)

Les Presses de l'Université de Montréal

ISSN

0316-0041 (imprimé)

1492-1367 (numérique)

Découvrir la revue

Citer cet article

Skordou, M. (2012). La judiciarisation des rébellions par les forces de l'ordre à Bruxelles de 1945 à 1975. Criminologie, 45(2), 277-299.

https://doi.org/10.7202/1013728ar
Résumé de l'article

Nous étudions la judiciarisation du phénomène rébellionnaire, en tant qu'ensemble d'événements isolés de violence contre des forces de l'ordre, à Bruxelles de 1945 à 1975. Nous avançons la thèse selon laquelle la judiciarisation du phénomène est dominée par l'action policière. Afin de l'appuyer, nous allons montrer que tant dans l'interaction des agents avec les citoyens que dans leur interaction avec les magistrats du parquet, les forces de l'ordre dominent la construction pénale des faits. Par ailleurs, nous soutenons que la mainmise policière dans la judiciarisation des rébellions relève de la dépendance professionnelle des magistrats du parquet des agents de l'ordre, hypothèse déjà soutenue par Grosman (1971). Nous illustrons, enfin, ce propos par un exemple issu de nos sources. 


\title{
La judiciarisation des rébellions par les forces de l'ordre à Bruxelles de 1945 à 1975
}

\author{
Melpomeni Skordou \\ Doctorante \\ École de criminologie, Université catholique de Louvain \\ melpomeni.skordou@uclouvain.be
}

RÉSUMÉ • Nous étudions la judiciarisation du phénomène rébellionnaire, en tant qu'ensemble d'événements isolés de violence contre des forces de l'ordre, à Bruxelles de 1945 à 1975. Nous avançons la thèse selon laquelle la judiciarisation du phénomène est dominée par l'action policière. Afin de l'appuyer, nous allons montrer que tant dans l'interaction des agents avec les citoyens que dans leur interaction avec les magistrats du parquet, les forces de l'ordre dominent la construction pénale des faits. Par ailleurs, nous soutenons que la mainmise policière dans la judiciarisation des rébellions relève de la dépendance professionnelle des magistrats du parquet des agents de l'ordre, hypothèse déjà soutenue par Grosman (1971). Nous illustrons, enfin, ce propos par un exemple issu de nos sources.

MOTS-CLÉS - Résistance à l'autorité policière, ministère public, Bruxelles, Trente Glorieuses.

\section{Introduction}

Cet article porte sur la judiciarisation des rébellions (art. 269, 271 à $274 \mathrm{du}$ Code pénal de 1867), des outrages (art. 275 à 277) et des faits de violence (art. 278 à 282) contre les policiers et gendarmes agissant dans le territoire administratif de Bruxelles de 1945 à 1975. Il constitue un volet d'une recherche ${ }^{1}$ plus vaste portant sur la répression du phé-

1. Action de recherche concertée «Jeunesse et violence en Belgique, 1880-2006: approches socio-historiques» sous la direction des promoteurs Fabienne Brion, Xavier Rousseaux et Jean-Michel Chaumont. Le projet a démarré le $1^{\text {er }}$ octobre 2007 et s'est clôturé le 31 septembre 2012 (Convention ARC Nº7/12-006). 
nomène rébellionnaire en Belgique (1880-1980) et plus particulièrement à Bruxelles (1945-1975).

Nos travaux précédents ont été consacrés au recensement de la littérature scientifique francophone et dans une moindre mesure anglosaxonne sur la question. À l'issue de ce dépouillement d'études, nous avons constaté l'attention particulière portée par la majorité des chercheurs sur une forme particulière de rébellion, celle que l'on peut qualifier de collective et juvénile (Skordou, 2010: 1117-1119, 2011 : 181-185). Nous avons aussi confronté la représentation scientifique de la rébellion à la construction qu'en donne la justice. Pour ce faire, nous avons étudié la répression du phénomène au moyen de l'analyse des statistiques judiciaires recensant l'activité du tribunal correctionnel de 1880 à 1980 (Skordou, 2010). L'observation de la criminalisation des rébellions sur tout un siècle a permis de relativiser l'évidence de leurs caractères collectif et juvénile; ceux-ci se révèlent moins universels quand on étudie l'objet dans des circonstances socio-politiques diverses. Nous avons encore établi (Skordou, 2012), selon l'exemple des travaux menés par Jobard (Jobard, 2006; Jobard et Névanen, 2007), le profiltype des conflits rébellionnaires ${ }^{2}$, produit de la répression du phénomène. Les dossiers des délits rébellionnaires jugés devant le tribunal correctionnel de Bruxelles pour la période de 1945 à 1975 ont été étudiés. Nous y reviendrons infra.

Dans cet article, nous avançons la thèse selon laquelle les forces de l'ordre (polices communales et gendarmerie nationale agissant dans le territoire administratif des 19 communes de Bruxelles) constituent la pierre angulaire de la construction pénale du phénomène rébellionnaire; ceci corrobore l'hypothèse de la dépendance professionnelle des magistrats du parquet des agents.

Pour le montrer, nous allons procéder en deux temps. D'abord, nous nous pencherons sur l'interaction entre les citoyens et les agents. Nous allons décrire les caractéristiques des conflits rébellionnaires en mettant en avant la banalité des conflits ainsi que l'initiative des agents de l'ordre dans l'éclatement des résistances. Dans un second temps, l'interaction entre agent et magistrat va nous intéresser. On s'attachera à

2. Le terme «IPDAP» qui signifie «infraction à personne dépositaire de l'autorité publique» est employé en France pour parler des rébellions, des outrages et des faits de violences contre les représentants de l'ordre et renvoie aux infractions à caractère rébellionnaire que nous traitons ici (contre policiers et gendarmes pour la Belgique des Trente glorieuses). 
l'étude des trois indicateurs suivants: la comparaison du taux de poursuite des délits de type rébellionnaire avec celui des violences interpersonnelles ${ }^{3}$; la façon dont la qualification policière d'un délit rébellionnaire est modifiée ou non par le magistrat du Ministère public; ainsi que le contenu des circulaires du procureur général concernant les délits en question.

Notre thèse, suggérant que dans le cas de la rébellion, ce n'est pas le procureur mais la police qui constitue la pierre angulaire de la judiciarisation du phénomène, peut paraître étonnante mais elle concorde avec l'hypothèse déjà évoquée par Grosman (1971: 263-264). Selon celle-ci, la dépendance professionnelle du magistrat du parquet à l'égard des policiers conditionne le travail du premier. Nous ajoutons que si cette dépendance n'existait pas, le fait que la victime des coups et blessures soit un policier ou un simple citoyen ne devrait pas avoir d'influence sur la façon de traiter l'affaire ${ }^{4}$. Le même type de violence avec un policier comme victime ou un non-policier comme victime devait être traité de la même manière. Or, ceci n'est pas le cas, comme nous allons voir dans la section Le policier et le procureur du Roi.

Nous allons clôturer ce travail par la description d'une affaire de coups et blessures volontaires qui se transforme en rébellion. Il s'agit d'un exemple qui illustre la vraisemblance de la thèse selon laquelle une interdépendance existe entre magistrats et policiers qui conduit à un traitement préférentiel de la victimisation de ces derniers. L'échantillon complet des dites affaires fera l'objet d'une prochaine étude.

3. Le mérite de cette comparaison sera décrit dans la section suivante.

4. Il n'est pas sans intérêt d'observer que selon le Code pénal belge de 1867 (art. 271) une rébellion (attaques ou résistances avec violences ou menaces) commise par une seule personne sans armes est punie par un emprisonnement de huit jours à six mois et (art. 398) les coups et blessures volontairement commis contre un particulier sont punis par un emprisonnement de huit jours à six mois également (et d'une amende de 26 à $100 \mathrm{frs}$ ). Dès lors, la loi (avec les peines comminées) ne préconise pas un traitement différentiel de ces contentieux. Le code pénal prévoit (art. 278) un emprisonnement plus sévère allant de deux mois à deux ans et une amende de cinquante à cinq cents francs pour celui qui aura frappé un policier ou gendarme en fonction; le mot «frapper» signifie «porter des coups» selon le Code pénal interprété par J. S. G. Nypels («Le code pénal belge interprété, principalement au point de vue de la pratique par ses motifs: la comparaison des nouveaux textes avec ceux du code de 1810 et pour les textes anciens conservés», Nypels, 1867). Néanmoins, il s'avère dans la pratique que l'article 281 n'est utilisé que dans $12,93 \%$ des peines. 


\section{Méthode}

Nous souhaitons montrer que l'action policière domine la judiciarisation des conflits rébellionnaires à Bruxelles de 1945 à 1975. Pour ce faire, nous nous concentrons sur l'action des trois acteurs: le citoyen, la police et le parquet. La relation des deux premiers nous intéresse car ce sont les protagonistes du conflit rébellionnaire, alors que la relation entre la police et le parquet nous importe car il s'agit des deux principaux acteurs du renvoi pénal. Si le rôle de la police dans la procédure pénale est bien connu (constatation et enregistrement des infractions, rédaction des procès-verbaux à l'intention du procureur du Roi, enquête judiciaire sous la direction de ce dernier...), le rôle du procureur du Roi mérite quelques précisions.

Le Ministère public (ou parquet ${ }^{5}$ ), dirigé par le procureur du Roi, est responsable de l'exercice de l'action publique; il reçoit des plaintes qui lui sont directement déposées par des particuliers ainsi que des procès-verbaux de la police ou la gendarmerie consignant des faits susceptibles de constituer des infractions pénales. Le procureur coordonne l'enquête de police judiciaire (phase de l'information) ou sollicite, si nécessaire, l'ouverture d'une instruction (phase de l'instruction pour des infractions complexes ou très graves pendant laquelle un juge d'instruction mène une enquête plus approfondie et décide de la suite de l'affaire). À l'issue de l'information, le procureur dicte la suite de l'affaire: cette dernière peut être classée sans suite ${ }^{6}$ quand l'opportunité de poursuivre fait défaut, pour des motifs divers et assez peu détaillés,

5. Jusqu'à la réforme de la carte judiciaire de 2012, la Belgique comptait 27 parquets, chacun alimentant les tribunaux d'un arrondissement judiciaire.

6. Le parquet «... a la possibilité de classer sans suite pour inopportunité des poursuites (les faits sont insignifiants, aucun antécédent chez le prévenu) et ce, même si les éléments de l'infraction sont établis et même s'il y a des charges suffisantes.» (Screvens, 1984-1985: 14). Ce pouvoir discrétionnaire confère au procureur un rôle très puissant dans l'administration de la justice pénale. van de Kerchove (1986) note que selon l'article $22 \mathrm{du}$ Code d'instruction criminelle napoléonien de 1808, toujours en vigueur en Belgique, «les procureurs du Roi sont chargés de la recherche et de la poursuite de tous les délits dont la connaissance appartient aux tribunaux correctionnels ou aux cours d'assises» (p. 80); ce qui semble imposer la règle inverse. Selon certains auteurs, et pour l'époque qui nous concerne (la Loi du 12 mars 1998 a inséré dans le Code d'instruction criminelle un article 28 quater dont le $\S 1$ reconnait explicitement que le procureur du Roi juge de l'opportunité des poursuites), ce serait sur la Loi du 9 août 1963 modifiant l'article 14 de la Loi du 15 juin 1935 sur l'emploi des langues en matière judiciaire que l'appréciation de l'opportunité des poursuites se base. La loi prévoit que «à la clôture de l'information en matière correctionnelle, le Ministère public, s'il ne classe pas l'affaire sans suite, transmet le dossier pour poursuites éventuelles [...].» (van de Kerchove, 1986: 81). 
et la procédure pénale reste alors suspendue jusqu'à l'apparition de nouvelles preuves; quand les éléments nécessaires à la poursuite sont présents, l'accusé peut être cité directement devant la juridiction adéquate pour son jugement.

Étant donné que le délit de rébellion ressemble fort à celui des violences interpersonnelles, sauf le statut différent des victimes, nous comparerons la judiciarisation des deux infractions lorsque cela sera nécessaire pour appuyer notre thèse.

La démonstration de notre thèse exige la mobilisation de plusieurs sources contenant des données différentes et produites par deux auteurs principaux : les agents de l'ordre (police et gendarmerie) et le procureur.

Concernant la police, le procès-verbal initial et final et l'ensemble des interrogatoires issus des dossiers jugés nous informent sur le conflit et ses circonstances (heure, lieu, nature de la violence), le profil du rebelle (âge, sexe, profession, état civil, usage d'alcool) et le rôle de la police dans l'éclatement du conflit et sa construction pénale (mode de saisine des agents et nombre et nature des chefs d'accusation).

À propos du parquet, la citation directe à comparaitre devant le tribunal correctionnel, issue des dossiers jugés, nous renseigne sur le nombre et la nature des qualifications pénales du procureur. Étant donné la pauvreté des dossiers quant aux informations relatives à l'activité du parquet, nous mobilisons deux sources supplémentaires. Le registre aux notices et les circulaires du parquet. La première source nous informe, parmi d'autres, sur le nombre des affaires de rébellion entrantes au Ministère public et sur le pourcentage de poursuites. L'étude des circulaires nous renseigne sur la politique criminelle pour les conflits en question.

Enfin, nous mobilisons la comparaison de la judiciarisation de la rébellion avec celle des violences interpersonnelles, telle qu'elle apparaît dans le travail de De Weirt (2011).

Comme le note Garnot (2006), l'étude des archives historiques présente deux grands intérêts. Le premier consiste à nous renseigner sur le fonctionnement du système pénal et sur le rôle de chaque agent dans l'administration de la justice. Le second repose sur «la mine inépuisable de renseignements qu'elles fournissent sur la société, les mentalités, la vie quotidienne, les comportements, la culture matérielle, l'économie (la liste n'est pas limitative)» (Garnot, 2006: 7).

Notre méthodologie s'inspire des travaux de Jobard (Jobard et Zimolag, 2005) et Deluermoz (2009) qui, contrairement à la majorité 
des recherches existantes, étudient les infractions contre les personnes dépositaires de l'autorité publique, en tant qu'événements isolés de violence contre les forces de l'ordre. Nous privilégions, comme le fait Deluermoz, une analyse interactionniste des sources judiciaires qui fait apparaître les interactions, entre citoyens et police et entre police et parquet, productrices d'un ordre public au quotidien. Ce faisant, on étudie les échanges directs entre les différents acteurs, qui prennent la forme de la dispute ou de l'échange de coups. Ces informations nous sont disponibles par l'étude qualitative des sources. Ensuite, différents éléments qui apparaissent de façon systématique dans chaque dossier (heure des faits, âge des acteurs, qualification du délit par la police et par le parquet, etc.) font l'objet d'un encodage et d'une analyse quantitative. Cela permet de mesurer certains éléments et de cerner les caractéristiques des protagonistes, des conflits et de leur judiciarisation. Nous pouvons maintenant examiner de plus près chacune des sources.

\section{Sources et limites}

\section{Les dossiers}

Les dossiers ${ }^{7}$ des personnes jugées par le tribunal correctionnel ${ }^{8}$ de Bruxelles, pour la période de 1945 à 1975, pour des faits d'agression (physique ou/et verbale) ou de résistance violente aux représentants de l'autorité représentent notre source la plus riche. Selon le Code pénal belge de 1867, les rébellions, les outrages et les violences aux agents constituent des délits devant être jugés devant le tribunal correctionnel. On mentionnera l'exception de la rébellion commise par plusieurs, avec armes et après concertation, qui est punissable de réclusion. C'est un phénomène très rarement judiciarisé (Skordou, 2010 : 1136) qui ne nous occupera pas ici.

Nous nous concentrons sur la judiciarisation des conflits banals et quotidiens. Nous étudions notamment les dossiers des condamnés ${ }^{9}$, volumineux en taille car ils tracent plusieurs étapes de la procédure

7. AEA (Archives de l'État à Anderlecht), TCB (Tribunal Correctionnel de Bruxelles), dossiers des affaires jugées de 1945 à 1975.

8. Voir Jobard et Zimolag (2005) où les auteurs analysent pour le cas français l'activité du tribunal correctionnel en s'intéressant à l'évolution de la répression des IPDAP, et aux discriminations observées selon la sociographie des rebelles.

9. Il y a vingt affaires qui comportent au moins un acquitté (Skordou, $2011: 187)$ : elles ne sont pas étudiées ici. 
pénale, qui témoignent des types de conflits à la fois ordinaires et assez dérangeants pour être pénalisés. Garnot (2006: 7) note l'intérêt d'étudier la banalité des contentieux au civil. Les affaires qui aboutissent au tribunal correctionnel ne sont pas moins banales (conflits répétitifs, violences réduites) et intéressantes à la fois: c'est justement cette banalité qui est très précieuse et qui permet de comprendre les caractéristiques des conflits quotidiens des individus ainsi que la façon dont la justice s'empare de ceux-ci.

L'état de la conservation des dossiers des affaires jugées est fortement défaillant ${ }^{10}$. En outre, le grand nombre des dossiers $\left(\mathrm{n} \approx 12000^{11}\right)$ ne permet pas une étude exhaustive. Nous avons dès lors procédé à un échantillonnage ${ }^{12}$ (Skordou, 2011 : 187-188) pour lequel la méthode de la collecte aléatoire des dossiers a été privilégiée, sachant que cette méthode exigeait d'atteindre un minimum de $250^{13}$ dossiers. Précisément, un corpus de 272 dossiers (soit 315 condamnés) a été constitué pour la période 1945-1975.

10. «... nous ne pouvons pas repérer les dossiers qui nous intéressent de manière systématique et homogène pour toute la période. Il s'avère difficile de suivre année par année l'évolution de la criminalisation du phénomène. Plus précisément, les trente ans étudiés ne sont pas conservés de manière uniforme ni en suivant la même logique et les mêmes règles. Ainsi, les dossiers de la période 1945-1963 semblent intégralement conservés sans pourtant être inventoriés de manière complète. Ensuite, la période 1964-1970 semble assez lacunaire étant donné que seulement les affaires d'un mois pour chaque année sont conservées. Enfin, pendant la troisième période qui s'étend de 1971 en 1975, toutes les affaires semblent avoir été conservées. Cependant, une logique de classement thématique soutient l'archivage de cette dernière sous-période du fonds: nous découvrons des boîtes qui ne concernent que des infractions contre l'ordre public et d'autres qui ne concernent que des vols, etc. » (Skordou, 2011 : 187-188).

11. La préservation inégale des dossiers rend leur exploitation épineuse. Il est impossible de connaître le nombre exact des dossiers de rébellions se trouvant dans l'ensemble des dossiers des affaires jugées en se basant sur la seule source des dossiers. Nous avons alors recouru à une autre source: les statistiques judiciaires publiées. Les statistiques nous permettent de calculer approximativement la taille $(\mathrm{N})$ de la population des dossiers de rébellion jugés devant le tribunal correctionnel de Bruxelles de 1945 à 1975.

12. Nous avons constitué un échantillon de dossiers avec la méthode de collecte aléatoire qui consiste à choisir des dossiers au hasard parmi tous les dossiers judiciaires conservés d'une période (en ne gardant que ceux qui concernent les types de délit en question) jusqu'à ce que la période soit équitablement couverte. Cette méthode devait nous permettre de construire un échantillon sans introduire de biais supplémentaires à ceux déjà imposés par la forme $\mathrm{du}$ fonds.

13. La taille de l'échantillon $n=250$ est choisie de façon à ce qu'une augmentation de «n» n'augmentera plus significativement la précision avec laquelle nous pouvons estimer une proportion «p» dans notre population (par exemple la part des femmes parmi les $\mathrm{N}$ cas). 
L'exploitation des dossiers des affaires jugées présente une limite: elle nous renseigne uniquement sur la nature des conflits poursuivis. Une comparaison entre ces derniers et les rébellions classées sans suite par le procureur (jusqu'il y a peu indisponibles et toujours non inventoriés) offrirait des indicateurs des motifs de la pénalisation d'un conflit. Pour pallier ce manque ainsi que pour d'autres motifs, nous avons exploité deux autres sources recensant l'activité du parquet: le registre aux notices et les circulaires du procureur général de Bruxelles.

\section{Le registre aux notices du parquet}

Le registre aux notices du parquet est une source sérielle et quantifiable disponible pour la période 1946 à $1966^{14}$. Elle témoigne du grand flux des infractions arrivant quotidiennement au parquet ainsi que de l'activité de tri et de sélection dont le procureur du Roi - et ses substituts - est l'auteur. Plus spécifiquement, s'y trouvent les informations concernant l'infraction (nom de l'auteur, type d'infraction, nom du plaignant, date de la plainte) ainsi que celles concernant l'activité des agents pénaux (service de la police avisé initialement, date d'arrivée au parquet, décision du procureur, et le cas échéant la date du jugement) ${ }^{15}$. Nous avons sondé le registre en commençant avec l'année 1947 et moyennant l'application d'un intervalle de cinq ans, nous avons terminé avec l'année 1966. Les affaires qui entrent au cours de la première semaine du mois de juin et de la première semaine du mois de novembre ont été analysées, afin de neutraliser un effet potentiel de la saison sur les faits (Skordou, 2012: 3).

\section{Les circulaires}

L'étude des circulaires du Ministère public pour la période 1945 à 1975 nous permet de faire certaines hypothèses concernant les stratégies du parquet quant aux questions d'ordre public. Ce fonds est entièrement publié en volumes annuels et conservé au centre de documentation du

14. Les registres de la première moitié de $20^{\mathrm{e}}$ siècle ont disparu, alors que ceux qui suivent l'année 1966 n'ont pas encore été versés au dépôt d'archives.

15. Le parquet de Bruxelles, le plus grand du royaume, est divisé en trois bureaux différents (quatre à partir de 1953 avec l'ajout du bureau R, responsable des infractions au code de la route): le bureau A (pour les infractions contre les biens), le bureau B, le seul qui nous intéresse ici (pour les infractions contre les personnes) et le bureau $\mathrm{F}$ (pour les infractions financières). 
parquet de Bruxelles. Chaque volume contient l'ensemble des circulaires que le procureur général a rédigées et adressées à divers destinataires (brigades de gendarmerie et commissariats de police, procureurs du Roi, juges d'instruction, et autres). Elles sont classées chronologiquement et une table de matières au début de chaque volume les répertorie par date en donnant leur thème. Nous les avons dépouillées en numérisant toutes celles qui étaient liées aux questions d'ordre public. De façon moins exhaustive, nous avons numérisé celles concernant la réglementation des situations de violence ayant un lien moins direct avec les conflits rébellionnaires.

\section{Une étude des poursuites en matière des coups et blessures volontaires}

Enfin, et dans le but d'évaluer la place que les conflits de type rébellionnaire occupent dans le système judiciaire et la priorité qu'ils constituent, nous comparons le traitement pénal qui leur est réservé avec celui réservé aux coups et blessures volontaires. L'étude de De Weirt (2011) sur les violences interpersonnelles à Bruxelles pendant les Trente Glorieuses nous a ainsi intéressée pour deux raisons: l'une méthodologique et l'autre substantielle. Tout d'abord, elle porte sur le même territoire et la même période que notre étude et elle se base sur les mêmes sources. Le délit des coups et blessures est jugé devant les mêmes juridictions que les rébellions. Ensuite, ses résultats montrent que les circonstances qui font naître les violences interpersonnelles sont de nature très similaire à celles provoquant les violences envers les représentants de l'ordre - à la différence des victimes. La comparaison de la répression réservée aux deux types d'infraction produit une meilleure compréhension du rôle de la police dans le système pénal bruxellois.

\section{La rébellion: une construction policière}

La judiciarisation de la rébellion s'opère à travers deux interactions principales. La première a lieu lorsqu'un agent de l'ordre, intervenant dans une querelle entre deux citoyens, rencontre une forme de résistance et décide de dresser un procès-verbal. La seconde se produit lorsque le procureur du Roi évalue l'opportunité de poursuivre pénalement le procès-verbal dudit agent. Nous suggérons que dans les deux cas, l'issue de l'interaction est dominée par la police. 


\section{Le policier et le rebelle}

Lorsque nous étudions les caractéristiques des interactions conflictuelles entre les citoyens et les forces de l'ordre, deux caractéristiques principales se dégagent: la banalité des circonstances violentes qui donnent naissance aux rébellions et le fait que c'est la police elle-même qui entraîne la résistance de la part des citoyens à la suite de son intrusion dans le règlement des conflits privés.

Tout d'abord, l'absence de tendance des citoyens à la révolte apparaît quand nous étudions le mode de saisine de la police lorsqu'elle déclenche le conflit rébellionnaire; dans $63 \%$ des cas, la police (ou la gendarmerie) intervient de façon proactive. Plus particulièrement, l'activité de patrouille des agents dans l'espace public de la ville est à la source de la moitié des conflits avec des citoyens; la permanence au commissariat $(6 \%)$, la régulation du trafic $(5 \%)$, ou le transport des prévenus du ou vers le palais de justice $(3 \%)$ sont également des situations au cours desquelles se produisent des résistances. Cette observation fait exception à une des lois de la sociologie policière que Zauberman (1982) formule de la façon suivante: «dans une forte majorité des affaires dont elle a connaissance, la police n'intervient pas de sa propre initiative, mais réagit à l'appel de tiers» (p. 23). Dans le cas de la rébellion bruxelloise de l'après-guerre, seulement $29 \%$ des affaires surviennent à la suite de l'appel d'un particulier (témoin ou victime). Par ailleurs, le fait que cette volonté prédéterminée d'agresser l'agent ou de résister à son action fait défaut découle de ce que la situation était violente déjà avant l'arrivée de la police. Des duels s'étaient formés avant son arrivée et c'est après son immixtion que la police se trouve en situation de victimisation.

Ensuite, les conjonctures qui font naître les rébellions et le profil social des protagonistes contrastent fortement avec la représentation classique que donne la littérature scientifique de ce phénomène (Skordou, 2010 : 1117-1119, 2011 : 181-185). Selon ces dernières, les conflits rébellionnaires sont issus des contextes d'agitation et contestation sociopolitiques où les jeunes se confrontent massivement et collectivement aux forces de l'ordre. Ceci a probablement été le cas en Belgique à la fin du $\mathrm{XIX}^{\mathrm{e}}$ et au début $\mathrm{du} \mathrm{Xx}^{\mathrm{e}}$ siècle. Toutefois, la réalité judiciaire de la rébellion bruxelloise de l'après-guerre se montre très différente.

Elle n'est pas une infraction collective. L'étude des dossiers confirme l'absence de résistance aux agents de l'ordre ou des violences commises collectivement. Dans plus de trois cas sur quatre, une seule personne résiste et est ensuite poursuivie (Skordou, 2011 : 193-195). 
Les protagonistes, dont $82 \%$ sont des hommes, ne sont pas jeunes: $61 \%$ sont âgés de 25 à 44 ans; ils sont mariés et installés professionnellement (Skordou, 2011: 189-193). Issus de la classe ouvrière ${ }^{16}$ et faiblement instruits, ils étaient impliqués dans une querelle d'ordre privé avant l'arrivée de la police.

La violence n'est pas grave. Les conflits sont d'une violence physique ou verbale faible et les raisons de la bagarre sont banales. L'infraction rébellionnaire la plus grave et la plus sévèrement punie par le Code pénal de 1867 (par la peine de réclusion réservée normalement aux crimes) est la rébellion armée commise par plusieurs et à la suite d'une concertation. Des 473 peines prononcées, seules 10 mobilisent l'article $272^{17}$ du Code pénal. Sur ces 10 condamnations, une seule affaire est caractérisée par l'usage d'armes. Ensuite, ce sont les coups qui causent une effusion de sang qui sont sévèrement punis, selon l'article $281^{18}$. Seulement $12,93 \%$ des peines prononcées mobilisent ledit article, sans pour autant que cela implique un emprisonnement supérieur à 6 mois, à l'exception de deux prévenus qui sont accusés en même temps pour rébellion et coups et blessures volontaires à un particulier.

La violence n'est pas issue d'un contexte d'agitation sociale. Elle caractérise, toutefois, un public avec un profil social ouvrier ${ }^{19}$, pour lequel la violence - ou ce que Schwartz (1990 : 299) appelle la libération des forces inbibées - présente une fonction sociale. En premier lieu, des comportements qui se caractérisent par ce déchargement des forces sont l'épuisement dans le travail physique, la consommation excessive d'alcool ou même la violence dans les relations avec les proches. Ensuite, et lorsque la violence s'expose au théâtre de la rue ${ }^{20}$ ou du bar ${ }^{21}$, elle peut aussi avoir une fonction de rétablissement de l'honneur et de la respectabilité masculine (Vrints, 2009). Ainsi, lorsque nous analysons

16. Pour l'analyse du profil social des rebelles voir Skordou (2012: 8-9).

17. Article 272 du Code pénal de 1867: Si la rébellion a été commise par plusieurs personnes, et par suite d'un concert préalable, les rebelles, porteurs d'armes, seront condamnés à la réclusion, et les autres à un emprisonnement d'un an à cinq ans. Si la rébellion n'a pas été le résultat d'un concert préalable, les coupables armés seront punis d'un emprisonnement d'un an à cinq ans, et les autres, d'un emprisonnement de trois mois à deux ans.

18. Article 281 du Code pénal de 1867 : Si les coups ont été la cause d'effusion de sang, de blessures ou de maladie, la peine sera un emprisonnement de trois mois à deux ans et une amende de 100 francs à 500 francs.

19. $40 \%$ exercent une activité professionnelle de type manuel et $23 \%$ sont des employés.

20. $62 \%$ des conflits éclatent dans la rue.

21. $21 \%$ des conflits éclatent dans les bistrots. 
les sites ${ }^{22}$ où les conflits éclatent, nous constatons une surreprésentation du centre de Bruxelles-ville ainsi que des communes urbanisées qui l'entourent, surtout habitées par les classes ouvrières à bas revenus. Les conflits surviennent dans les rues et les bistrots, tard dans la nuit et la consommation d'alcool est très souvent importante et conditionne les échanges d'insultes et de coups. L'usage de l'espace public par cette population s'explique aussi par le fait qu'elle ne dispose pas d'un espace privé propice au repos ou au loisir (Poncela, 2010: 18). À partir des années 1970 , la condition ouvrière s'améliore grâce à un meilleur accès aux biens de consommation. L'espace privé pourra être embelli et investi aussi pour les loisirs. Cependant, les travailleurs de l'industrie lourde n'auront pas l'énergie de s'y consacrer (Delcourt et Lamarque, 1960: 66).

Enfin, la violence privée qui donne naissance à la rébellion n'invite pas à la répression à cause de sa gravité mais plutôt en raison de sa visibilité. Alors que les protagonistes souhaitent régler leurs affaires sans l'intervention des agents de l'ordre, ces derniers s'y impliquent et rencontrent de la résistance. Défier l'autorité de l'agent publiquement est punissable même si ce comportement constitue un dommage collatéral. C'est un double tort, contre les valeurs que l'agent représente: l'honneur masculin et l'autorité. La banalité des circonstances faisant naître les rébellions et l'insignifiance de la violence contre les agents ne semblent pas empêcher la judiciarisation des faits. Enfin, la spécificité de la rébellion semble liée non pas aux caractéristiques des rebelles mais à celles des victimes qui sont en même temps les verbalisants.

\section{Le policier et le procureur du Roi}

Deux étapes sont décisives dans la judiciarisation de la rébellion: la constitution d'un procès-verbal par le représentant de l'ordre et la décision du parquet de poursuivre. Le policier et le magistrat constituent deux acteurs clés dans la judiciarisation du phénomène. Toutefois, nous suggérons que le rôle de la police est davantage déterminant que celui du parquet. Examinons trois indicateurs qui appuient notre thèse.

Premièrement, nous examinons le taux de poursuite des délits rébellionnaires, en nous limitant à la période 1946 à 1966 pour laquelle des archives sont disponibles. Ce taux, qui atteint en moyenne $50 \%$ (avec

22. Pour une étude géographique du phénomène rébellionnaire voir Skordou (2012: $4-8)$. 
des valeurs extrêmes de $37 \%$ en 1952 et de $64 \%$ à la fin des années 1950), s'avère très élevé, lorsqu'on l'étudie comparativement.

Nous le comparons notamment avec le recours au classement que mobilise le parquet de Bruxelles dans la longue durée, toutes infractions confondues. Les observations de Janssen (1991 : 322) témoignent d'une nette augmentation du classement qui passe le cap de $51,70 \%$ déjà en 1926 et arrive à 70,36\% en 1982. Selon la thèse de Davidovitch et Boudon (1964: 206), que Janssen ${ }^{23}$ (1992: 122) rejoint totalement, le fondement de cette pratique de classement se trouve dans la tendance du système répressif à réduire la part des solutions judiciaires coûteuses face à une augmentation pressante des affaires criminelles. Dans ce système judiciaire qui, à la sortie de la Seconde Guerre mondiale, privilégie de plus en plus le classement sans suite, le privilège (relatif) donné à la poursuite des conflits rébellionnaires n'est pas sans importance.

Cet argument n'appuie pas à lui seul la thèse du rôle central de la police dans la poursuite des faits rébellionnaires. À cette fin, nous devons recourir à une comparaison avec le taux de poursuite réservé aux coups et blessures volontaires (voir tableau 1). De Weirt (2011) analyse les registres ${ }^{24}$ aux notices du parquet pour les violences interpersonnelles et dévoile que le taux de poursuite fluctue de $7 \%$ en 1950 à $11,3 \%$ en 1964. Ce taux de conflits que le procureur renvoie devant le tribunal est beaucoup plus faible que celui des rébellions qui peut dépasser les $50 \%$. Cette comparaison est légitime et en même temps révélatrice en raison de la ressemblance entre les deux délits. Ressemblance quant aux caractéristiques de la violence mais aussi en ce qui concerne celles des auteurs. De Weirt a étudié ${ }^{25}$ la judiciarisation des violences interpersonnelles et il a créé une typologie des affaires. Selon celle-ci, les bagarres sur la voie publique et dans les bars constituent les phénomènes les plus recensés, et s'accompagnent d'une consommation d'alcool très prégnante dans les deux cas. Selon le profil social des prévenus ${ }^{26}$ que l'auteur a pu construire, il s'agit presque exclusivement d'hommes;

23. Pour une étude du classement sans suite en Belgique voir Janssen et Vervaele (1990).

24. De Weirt (2011) a sondé «différents registres du parquet pour les seuls mois de janvier des années 1950, 1958, 1959 et 1964.» (p. 221).

25. Son analyse porte sur le même territoire et période que notre étude et se base sur les mêmes sources: les dossiers des affaires jugées devant le tribunal correctionnel.

26. L'auteur a limité son étude aux violences impliquant des jeunes adultes âgés de 18 à 25 ans. 
parmi eux $30 \%$ sont mariés (malgré leur jeune âge); ils exercent des emplois «aux statuts modestes au sein de la société bruxelloise» (De Weirt, 2011 : 225). Cette image est semblable à celle qu'on obtient en étudiant le phénomène rébellionnaire. Enfin, les deux types de conflits impliquent des publics identiques et sont issus de conjonctures semblables à la différence que, dans le cas des violences rébellionnaires, les agents de l'ordre sont victimisés. Cette différence est importante et elle expliquerait le taux de poursuite très élevé du phénomène en question. Le Ministère public semble plus sensible à la revendication du statut de la victime de la part des agents de l'ordre qu'à la violence même des conflits. Le rôle central de la police dans la construction pénale de la rébellion se distingue.

TABLEAU 1

Taux de poursuite des rébellions et des coups et blessures volontaires

\begin{tabular}{|l|c|c|c|c|}
\hline & 1947 & 1957 & 1961 & 1966 \\
\hline $\begin{array}{l}\text { Rébellions, outrages et faits de violence } \\
\text { à agent (art. 269 à 282) }\end{array}$ & $46 \%$ & $64 \%$ & $57 \%$ & $46 \%$ \\
\hline $\begin{array}{l}\text { Coups et blessures volontaires } \\
\text { (art. 392, 398 à 401, 410) }\end{array}$ & $\begin{array}{c}\text { Janvier } \\
1950\end{array}$ & $\begin{array}{c}\text { Janvier } \\
1958\end{array}$ & $\begin{array}{c}\text { Janvier } \\
1959\end{array}$ & $\begin{array}{c}\text { Janvier } \\
1964\end{array}$ \\
\hline
\end{tabular}

Deuxièmement, pour questionner le rôle central de la police dans la construction pénale de la rébellion, nous nous intéressons à la qualification pénale qu'elle attribue à un délit rébellionnaire et à la façon dont cette qualification est modifiée ou non par le procureur. Cousineau et Cucumel (1991) observent en effet, au Canada, toutes infractions confondues, que dans $84,4 \%$ des cas, «le libellé des accusations enregistrées par les policiers au niveau de la demande d'intenter des procédures correspond exactement à celui retenu par les procureurs de la Couronne au moment de formuler définitivement l'acte d'accusation» (p. 70). En ce qui concerne les rébellions bruxelloises, nous étudions les procès-verbaux initiaux et finaux de la police ou la gendarmerie et la citation à comparaître devant le tribunal du procureur du Roi pour 
les 315 individus condamnés pendant la période 1945-1975. Ces documents, issus des deux acteurs de renvoi, recensent le nombre et la nature des chefs d'accusation ${ }^{27}$ et nous permettent de les comparer afin de repérer les éventuelles requalifications opérées par le magistrat du parquet.

L'étude des dossiers montre que, dans $80 \%$ de ceux-ci, le procèsverbal de police mentionne une à trois accusations ${ }^{28}$. Lorsqu'une seule accusation est retenue, il s'agit majoritairement du délit d'outrages (art. 276, Code pénal belge de 1867) (à l'exception de quelques rébellions, en référence à l'article 269). L'accusation du délit d'outrages est associée à celle de rébellion, ou de coups aux agents (art. 280). L'ivresse publique ou la violence interpersonnelle (art. 392, Arrêté-loi du 14 novembre 1939) accompagnent éventuellement le délit d'outrages à la police ou de rébellion. Enfin, quand trois accusations figurent au procès-verbal, les trois délits de type rébellionnaire sont majoritairement combinés avec l'ivresse publique ou avec les coups et blessures volontaires. Nous constatons que la configuration des diverses incriminations se rapporte au même événement: il ne s'agit pas «d'une accumulation de délits de même nature que l'on choisit de regrouper en un seul dossier [...] pour les traiter simultanément» (Cousineau et Cucumel, 1991: 66).

Ensuite, le procureur du Roi (et le juge du fond) adopte comme telles les accusations enregistrées par la police lorsqu'elles concernent la rébellion, les outrages et les violences contre les agents et, lorsqu'elles sont également constatées, l'ivresse publique ou la conduite de véhicule sous l'influence de l'alcool. Pour un ensemble de 315 accusés pour des délits rébellionnaires, le procureur requalifie très peu les infractions choisies par la police. Ainsi, quatre accusations pour menaces de mort à la police disparaissent, une cinquième se transforme en outrages à la police et dans un seul cas cette qualification est ajoutée par le magistrat. Par ailleurs, trois accusations policières d'attroupement sont abandonnées. En ce qui concerne les infractions connexes aux résistances à la police, elles sont majoritairement abandonnées. En effet, le refus d'exhiber sa carte d'identité est abandonné dans $95 \%$ des cas, le tapage nocturne est abandonné dans $72 \%$ des cas et l'infraction à un règlement de police est évacuée dans $83 \%$ des cas.

27. Voir Cousineau et Cucumel (1991: 60). Les auteurs se sont penchés plus précisément sur la question de l' «over charging» pour le cas canadien.

28. Dans cette partie des dossiers, $34 \%$ des individus sont accusés d'un délit, $25 \%$ de deux délits et $21 \%$ de trois délits. 
À cette étape quelques constats s'imposent. Quand le policier qualifie un comportement de rébellionnaire, cette qualification est respectée par le magistrat du parquet. Plus particulièrement, le nombre des chefs d'accusation et la nature de ceux-ci ne sont pas altérés. Au contraire, les délits connexes aux rébellions sont effacés presque automatiquement par le procureur. Non seulement la priorité de la répression des rébellions est confirmée mais une harmonie est également observée entre police et parquet. La qualification policière concorde avec celle du procureur qui ne la corrige que rarement. Ceci donne à la police un poids majeur dans la construction du phénomène rébellionnaire.

Troisièmement, nous étudions les circulaires émanant du procureur général de Bruxelles pour la période 1945 à 1975 afin d'examiner si le Ministère public exerce une politique criminelle spécifique en matière des délits rébellionnaires. Nous les dépouillons systématiquement et nous constatons une totale absence de circulaires réglementant les conflits en question. Les circulaires s'adressent aux commissariats, brigades et procureurs du Roi en réglementant (parmi d'autres) des différends concernant le roulage, le divorce et l'adultère, ou encore les infractions liées à l'ivresse. Ils introduisent des nouveaux modes de constitution des procès-verbaux ou encouragent les magistrats à donner priorité à leurs relations avec les membres du barreau. L'absence de circulaires qui traceraient une ligne préférentielle de politique concernant les rébellions confirme que la liberté d'action des magistrats (toujours dans les limites de la loi) n'est contrainte que par le bas. En exerçant son pouvoir discrétionnaire en matière de rébellion, le procureur s'aligne sur les informations policières et révèle le rôle central de la police dans la pénalisation desdits faits. Comme van de Kerchove (1986) l'écrit, l'existence du pouvoir discrétionnaire du parquet révèle l'existence des types de régulations moins formels que la loi mais qui produisent des effets aussi réels que la loi.

\section{Une hypothèse explicative}

Le rôle central de la police dans la construction pénale de la rébellion transparaît donc de l'association de trois indicateurs: le procureur classe de façon beaucoup moins importante (en comparaison du taux de classement des violences interpersonnelles) les procès-verbaux de rébellion; il respecte de très près les qualifications policières desdits délits; et ces conduites ne résultent pas d'une injonction de politique criminelle exprimée hiérarchiquement (par le procureur général). 
La mainmise des forces policières sur l'espace pénal belge et la limitation du Ministère public à un rôle passif de récepteur ont déjà été mises en avant par Janssen (1992: 140). Les magistrats du parquet ne sont pas avertis rapidement des affaires à traiter et reçoivent aussi des enquêtes policières lacunaires. Ils ne demandent pas pour autant à la police des recherches supplémentaires, classent rapidement et se limitent à un contrôle symbolique de la légalité de la phase policière. La police agit dès lors avec une grande liberté que le Ministère public balise ou contraint médiocrement malgré le rôle central qu'on lui attribue dans l'action pénale. Cousineau et Cucumel (1991: 71) ajoutent que la charge croissante des affaires à traiter amènerait les magistrats à adopter les évaluations policières. Que la charge soit croissante ou non, Grosman insistait déjà en 1971 sur l'impossibilité matérielle de contrôler l'information fournie par la police. «C'est la police qui rédige la feuille incriminante», déclare un procureur, «c'est la base de notre poursuite. Nous ne vérifions pas l'information, car s'il fallait la contrôler il nous faudrait deux fois plus de procureurs à la cour...» (Grosman, 1971 : 263).

Zauberman (1982) suggère que le succès des policiers en tant que renvoyants pénaux est dû «d'abord au fait qu'ils ont un accès direct, formalisé au système judiciaire» (p. 34). Ceci explique d'ailleurs le pouvoir, certes officieux, des agents de l'ordre de déjudiciariser les faits qui - selon leur connaissance de la politique criminelle du Ministère public - seraient classés une étape plus loin ${ }^{29}$. Cousineau et Cucumel (1991: 71) questionnent la véritable convergence entre le libellé des accusations enregistrées par la police et celui retenu par les procureurs. Les auteurs évoquent aussi la capacité d'une agence de contrôle comme la police d'anticiper ce qu'elle pense être la politique criminelle du Ministère public.

Le succès des policiers est dû aussi «à ce que leurs fonctions les font bénéficier d'une présomption de compétence» (Zauberman, 1982: 38). Grosman (1971) exprime cette présomption en termes de confiance: «Le rôle du procureur est subordonné à celui de la police, il s'établit entre les deux un courant de confiance réciproque» (p. 264).

En matière de rébellion, ces explications restent pertinentes, mais nous pouvons souligner spécifiquement le poids de la dépendance entre magistrats et policiers. Dans les nombreuses affaires de violence ordi-

29. Concernant la capacité d'une agence d'anticiper ce qu'elle pense être la réaction possible des étapes ultérieures voir Robert (1977: 13). 
naire, jugées devant le tribunal correctionnel, les déclarations consignées dans les procès-verbaux de police semblent validées par les magistrats. Lorsque le verbalisant est la victime (prétendue), le magistrat voit sa «passivité confiante» s'accroître car le classement sans suite ou la requalification à la baisse représenterait un camouflet à la relation de coopération organique et nécessaire entre collaborateurs de justice.

L'hypothèse supplémentaire de la dépendance permet de rendre compte du traitement différentiel de la victimisation des agents et de celle de simples citoyens. Plus particulièrement, si l'hypothèse de la dépendance n'était pas vraie, le traitement pénal réservé par le parquet aux coups et blessures dont les policiers sont victimes devrait être identique au traitement accordé aux coups et blessures infligés à n'importe quel citoyen. Ni la loi (avec les peines comminées ${ }^{30}$ ) ni les circulaires (avec leurs priorités en matière de poursuites) ne préconisent un traitement différentiel de ces contentieux.

Afin d'illustrer par un exemple le traitement pénal préférentiel de la victimisation des policiers par le parquet, nous relaterons les détails d'une affaire. Il s'agit d'un dossier pénal de violence conjugale, contexte qui est évacué pour faire place à un outrage à la police. Plus particulièrement, Jeanine est maltraitée physiquement et verbalement presque quotidiennement par Charles, son époux. À partir de février 1960, elle se rend une fois par mois à la police afin de porter plainte pour violences volontaires. Les époux sont chaque fois appelés à témoigner. Les procès-verbaux contiennent les témoignages des deux époux et la même version des histoires de violence se confirme: Jeanine est, de façon systématique, victime de violence conjugale.

Lorsque je rentre chez moi, mon épouse est très souvent absente. À son retour, cela provoque des discussions au cours desquelles elle me provoque. C'est ainsi que je suis obligé de la repousser et qu'elle heurte des meubles ou qu'elle contusionne. Elle prétend alors que je la bats. Il est exact qu'au cours des scènes, il m'est arrivé de déchirer ses vêtements de colère et de la gifler. Elle est extrêmement nerveuse et n'est pas à même de diriger son ménage. [...]. Il m'arrive, au cours des disputes, de dire que je ferai «crever ma femme petit-à-petit». Je veillerai à ne plus la battre mais elle est très provocante ${ }^{31}$.

30. Voir la note 4 .

31. Anderlecht, Archives de l'État (AEA), Fond du tribunal correctionnel de Bruxelles, affaires jugées, $\mathrm{n}^{\circ}$ greffe 6257, jugement du 22 septembre 1962. 
Malgré l'évidence de la situation problématique qui se perpétue dans ce ménage, aucun des cinq procès-verbaux successifs de la police ne justifie un renvoi pénal de Charles devant le tribunal correctionnel. Toutefois, en novembre de la même année, Charles est arrêté par des policiers en patrouille pour avoir agressé un passant. Sa femme, présente lors de cette scène, insulte les policiers qui sont en train d'embarquer son époux pour l'amener au commissariat. Issue de l'affaire: Charles est condamné à 1 mois et 8 jours d'emprisonnement pour violences exercées contre un passant et Jeanine est condamnée à 8 jours d'emprisonnement et 26 francs d'amende pour outrage à la police. Il semble que la violence publique contre les représentants de l'autorité est intolérable. Même si c'est une violence minime, en comparaison de la violence conjugale, grave et répétitive, elle est directement renvoyée par le magistrat devant le tribunal (et sanctionnée par le juge). Même quand l'échelle de la violence est beaucoup moindre dans le cas des policiers, le fait de porter atteinte à leur autorité en public constitue un affront intolérable. Dans une société où l'honneur est une qualité à préserver, être homme et représentant de l'autorité semble «doubler» la gravité de l'insulte en public. En même temps, le policier a aussi par son arrestation «les mains qui salit $[\text { sic }]^{32} »$ et les outrages témoignent non seulement de l'inefficacité de son autorité mais aussi d'une tentative du rebelle de la renverser:

«[...] si, lors de leur passage [celui des policiers], les voix tendent à diminuer et les gestes à se faire discrets, une des formes de résistance à cette influence consiste à faire exactement le contraire ${ }^{33} »$. La violence privée dans la discrétion du foyer et la plainte d'une femme battue ne font pas, au contraire, l'objet de l'attention du magistrat.

\section{Conclusion}

Dans une interaction qui tourne mal entre un citoyen et un agent de l'ordre, ce dernier semble disposer de plus de moyens pour imposer sa vérité. L'agent possède un monopole du récit des faits, susceptible de produire une réaction pénale favorable ou défavorable à l'accusé. L'événement - le problème - devient donc «manipulable» (Monjardet,

32. Deluermoz (2009): «Selon une insulte courante dans les quartiers populaires, l'agent a "les mains qui salit [sic]": elles salissent en effet les identités et les réputations.» (p. 443).

33. Loc.cit. 
1985 : 303) par le policier. Nous l'avons constaté, nos rebelles ne sont pas si rebelles que cela après tout. Ils font usage de l'espace de la rue et du café tant pour leur plaisir de boire que pour régler leurs comptes, avec bruit et fureur. Cet usage de la violence physique et verbale relève d'un déchargement des forces et de l'affirmation d'une masculinité populaire. Pour l'agent qui cherche à y mettre de l'ordre et qui rencontre de la résistance ou subit de la violence, il s'agit clairement d'un manque de respect à l'égard de l'autorité et d'une atteinte à son honneur. Le policier étant à la fois la victime et celui qui relate officiellement sa victimisation auprès de l'autoritée ${ }^{34}$, son influence ${ }^{35}$ n'est pas sans importance.

La judiciarisation de la rébellion met en scène un équilibre spécifique des forces respectives de la police et du ministère public.

La police se trouvant en amont du système pénal, à l'entrée de l'entonnoir, son action de filtrage est très puissante et ceci pour deux raisons principales: d'abord, exception faite des affaires urgentes qui sont traitées immédiatement, l'agent accorde une priorité différente à chaque affaire selon divers critères ${ }^{36}$, qui ne relèvent pas seulement de la loi mais bien d'une multitude de facteurs que rappellent De Valkeneer et Francis (2007). Ensuite, une fois qu'un fait susceptible de constituer un délit devient l'objet d'un procès-verbal, c'est ce dernier qui constitue le seul rapport officiel des faits, la définition dominante de la situation.

Le parquet est considéré comme la pierre angulaire du système pénal belge. Il vérifie la légalité de la phase policière, il coordonne l'information et évalue l'opportunité de poursuivre les infractions dont il a connaissance. Son pouvoir discrétionnaire, même s'il est d'origine coutumière, lui accorde un pouvoir très important. Cependant, ce pouvoir semble s'affaiblir devant celui du policier, spécifiquement dans le cas de la rébellion.

La qualification pénale que l'agent donne à une résistance policière est adoptée comme telle par le parquet, sans quaucune politique criminelle l'exige. Qui plus est, dans plus de la moitié des cas, ces affaires sont directement renvoyées devant le juge du fond. Le pourcentage est significatif dans un système judiciaire qui fait un usage de plus en plus important du classement sans suite au fil du temps (Janssen, 1991). De

34. Voir aussi Jobard et Névanen (2007): «Par essence, ces infractions sont les seules dont les agents qui les constatent sont également ceux qui s'en disent victimes.» (p. 244).

35. Qu'il exerce son influence sur la poursuite par «manipulation» du magistrat (Lévy, 1985) ou dans un esprit coopératif (Mouhanna, 2004), n'est pas l'objet de notre analyse.

36. Concernant l'autonomie policière, voir De Valkeneer et Francis (2007: 21-25). 
surcroît, la comparaison avec la pratique du parquet face aux violences interpersonnelles, avec un taux moyen de classement sans suite de $90 \%$ pour la période 1946 à 1966, démontre que la police constitue la clé de voûte de la construction du phénomène rébellionnaire ${ }^{37}$.

\section{Références}

Cousineau, M. M., \& Cucumel, G. (1991). De la police au tribunal: formulation et cheminement des plaintes portées devant la Cour du Québec, Chambre criminelle et pénale. Criminologie, 24 (2), 57-79.

Davidovitch, A., \& Boudon, R. (1964). Les mécanismes sociaux des abandons de poursuite. Analyse expérimentale par simulation. L'année sociologique, 3, 111-244.

Delcourt, J., \& Lamarque, G. (1960). Un faux dilemme. Embourgeoisement ou prolétarisation de la classe ouvrière. Bruxelles: La pensée catholique.

Deluermoz, Q. (2009). Présences d'État. Police et société à Paris (1854-1880). Annales. Histoires, Sciences sociales, $64^{\mathrm{e}}$ année, 435-460.

De Valkeneer, C., \& Francis, V. (2007). Manuel des sociologies policières. Bruxelles: Larcier.

De Weirt, X. (2011). Des jeunes adultes jugés devant le tribunal correctionnel de Bruxelles. La perception des comportements violents entre rouages judiciaires et approche de la réalité (1946-1975). In X. Rousseaux, \& X., De Weirt, X. (eds.), Ville, violence et jeunesse en Europe: une approche socio-bistorique du Moyen Âge à nos jours (209-239). Louvain-la-Neuve: Presses universitaires de Louvain.

Garnot, B. (dir.). (2006). La justice et l'bistoire. Sources judiciaires à l'époque moderne (XVIe, XVIIe, XVIIIe siècles). Rosny-sous-Bois: Éditions Bréal.

Grosman, B. A. (1971). Le procureur de la poursuite criminelle: étude sur l'exercice du pouvoir de discrétion. Les cabiers de droit, 12 (2), 241-286.

Janssen, C. (1991). La politique de classement sans suite du ministère public en Belgique, 1836-1982. Histoire $\mathfrak{G}$ Mesure, 6 (3), 313-347.

Janssen, C. (1992). Police et ministère public, du malaise à la réflexion et aux propositions de réforme. Déviance et Société, 16 (2), 117-141.

Janssen, C., \& Vervaele, J. (1990). Le Ministère public et la politique de classement sans suite. Bruxelles: Publications du Centre national de criminologie (Vol. 14), Bruylant.

37. Comme un lecteur remarque, les agents, du fait de leur accès direct au système judiciaire, sont des victimes davantage susceptibles de témoigner devant le tribunal et de soutenir l'issue pénale de l'affaire. Toutefois, la victime d'une violence interpersonnelle ne joue pas toujours un rôle actif dans la poursuite (en se constituant partie civile, en se faisant représenter par un avocat) ce qui pourrait inciter certains procureurs de classer un nombre d'affaires. N'ayant pas assez d'éléments pour traiter cette question, on y reviendra dans une prochaine étude. 
Jobard, F. (2006). La racaille en politique, enquêtes sur les jeunes «connus des services de police «. Vacarme, 35. Consulté le 19 juillet 2009, http://www. vacarme.org/article512.html.

Jobard, F., \& Névanen, S. (2007). La couleur du jugement. Discriminations dans les décisions judiciaires en matière d'infractions à agents de la force publique (1965-2005). Revue française de sociologie, 48 (2), 243-272.

Jobard, F., \& Zimolag, M. (2005). Quand les policiers vont au tribunal. Étude sur les outrages, rébellions et violences à agents. Questions pénales, 18 (2), $1-4$.

Lévy, R. (1985). Scripta manent: la rédaction des procès-verbaux de police. Sociologie du travail, 4, 408-423.

Monjardet, D. (1985). Police et sociologie: questions croisées. Déviance et société, 9 (4), 297-311.

Mouhanna, M. (2004). Les relations police-parquet en France: un partenariat mis en cause? Droit et société, 3 (58), 505-520.

Nypels, J. S. G. (1867). Le Code pénal belge interprété, principalement au point de vue de la pratique par ses motifs: la comparaison des nouveaux textes avec ceux du code de 1810 et pour les textes anciens conservés. Bruxelles: Bruylant.

Poncela P. (2010). La pénalisation des comportements dans l'espace public. Archives de politique criminelle, 32, 1-21.

Robert, P. (1977). Les statistiques criminelles et la recherche. Réflexions conceptuelles. Déviance et société, 1 (1), 3-27.

Schwartz, O. (1990). Le monde privé des ouvriers. Hommes et femmes du Nord. Paris: Presses universitaires de France.

Screvens, R. (1984-1985). Notes du cours de procédure pénale comparée. Bruxelles: Presses universitaires de Bruxelles.

Skordou, M. (2010). Les infractions contre l'ordre public en Belgique de 1880 à 1980 : les statistiques judiciaires au service de la déconstruction d'un objet d'étude. Revue de droit pénal et de criminologie, 11, 1117-1150.

Skordou, M. (2011). La rébellion à Bruxelles après la Seconde Guerre mondiale (1945-1975). Un contre-exemple à la construction sociale dominante du phénomène comme action collective et juvénile. In X. Rousseaux, \& X., De Weirt (ed.), Ville, violence et jeunesse en Europe: une approche socio-bistorique du Moyen Age à nos jours (181-207). Louvain-la-Neuve: Presses universitaires de Louvain.

Skordou, M. (2012). Résistances à l'autorité policière à Bruxelles (1945-1975): la construction judiciaire de la violence populaire. Brussels Studies, 59, 1-13.

van de Kerchove, M. (1986). Fondement et limites du pouvoir discrétionnaire du ministère public. Aux confins de la légalité. Sociologie et sociétés, 18 (1), 77-96.

Vrints, A. (2009). All honourable men? Violence and manliness in twentiethcentury Antwerp. Sextant, 27, 89-101.

Zauberman, R. (1982). Renvoyants et renvoyés. Déviance et société, 6 (1), 23-52. 


\section{Sources}

Le Code pénal belge (1867).

Le Code d'instruction criminelle belge (1808).

\section{Lois belges}

Article 14 de la Loi du 15 juin 1935, concernant l'emploi des langues en matière judiciaire.

Articles 1 et 14 de l'Arrêté-loi du 14 novembre 1939 relatif à la répression de l'ivresse publique, pris en exécution de la loi du 7 septembre 1939.

Loi du 12 mars 1998, concernant l'opportunité des poursuites du procureur du Roi.

\section{Archives judiciaires de 1'État}

Archives judiciaires de l'État, Parquet de Bruxelles, Registres aux notices, 19461966. Parquet de Bruxelles

Archives judiciaires de l'État, Tribunal de première instance de BruxellesCorrectionnel, affaires jugées, 1945-1975.

ABSTRACT - We study the judiciarisation of resistance to police authority in Brussels from 1945 to 1975, a phenomenon consisting of a certain number of isolated cases of violence against police and constabulary agents. We suggest that the construction of the resistance to police authority as a judicial phenomenon is dominated by police agents. In order to corroborate our thesis we examine police interactions with citizens as well as with public prosecutors and show that in both cases the agents constitute the cornerstone of the judicial construction of the phenomenon. This thesis supports the hypothesis of the magistrates' professional dependence on the police agents, which has already been proposed by Grosman (1971). We finally illustrate our thesis by a specific example of a violence-against-police-authority case, taken from our sources.

KEYWORDS - Resistance to police authority, public prosecutor, Brussels, Glorious Thirty.

RESUMEN - Nuestro estudio trata la judicialización de la resistencia a la autoridad policial en lo que concierne a hechos aislados de violencia contra las fuerzas del orden, acaecidos en Bruselas entre 1945 y 1975. Nuestra hipótesis sugiere que la judicialización de este fenómeno está dominado por la acción policial. Con el fin de sustentar dicha hipótesis, demostraremos que, tanto en la interacción entre los agentes y los ciudadanos como en aquella entre los agentes y los jueces, las fuerzas del orden influyen en gran medida en la construcción penal de los hechos. Además, sostenemos que ésta influencia policial, en lo que refiere a la judicialización de las rebeliones, revela la dependencia profesional de los jueces hacia las fuerzas del orden, hipótesis que ha sido sustentada por Grosman (1971). Finalmente, ilustraremos dicha hipótesis citando un ejemplo de nuestras fuentes.

PALABRAS CLAVE - Resistencia a la autoridad policial, ministerio público, Bruselas, Gloriosos Treinta. 